VISUAL EXPERIMENT

\title{
Experimental strategy of animal trial for the approval of anti- diabetic agents prior to their use in pre-human clinical trials
}

\author{
Vivek K. Bajpai, Irfan A. Rather and Gyeong-Jun Nam \\ Department of Applied Microbiology and Biotechnology, Yeungnam University, Gyeongsan 712-749, South \\ Korea.
}

First two authors contributed equally

Correspondence to Vivek K. Bajpai at vbiotech04@gmail.com Fax: +82-53-813-4620

First Published: 16 December, 2015; Updated version: 1.0

DOI: 10.3329/bjp.v11i1.25762

\section{ABSTRACT}

Although several naturally available drugs have been historically used for the treatment of diabetes mellitus throughout the world, few of them have been validated by scientific criteria. Before approval of any drug developed it should pass through animal trial prior to clinical human trial, which should followed by some standard ethical rules. Recently, a large diversity of animal models have been developed to better understand the pathogenesis of diabetes mellitus, and new drugs have been introduced in the market to treat this autoimmune disease. In the present article, we demonstrated some standard handling procedure of animal trial for the approval of anti-diabetic drug, which could be helpful for both academics and industrial scientific community to conduct the animal experiments. This research also contributes in the field of ethnopharmacology to design new strategies for the development of novel drugs to treat this serious condition of diabetes mellitus that constitutes a global public health.

\section{INTRODUCTION}

The increasing worldwide incidence of diabetes mellitus in adults constitutes a global public health burden. There is also a great interest in the development of new drugs to prevent the burden of complications associated with this disease and the raised interest in the scientific community to evaluate drug/ products in experimental studies in animal models and also few of them were tested in humans (Frode and Medeiros, 2008). Practically, any newly developed anti-diabetic drug before its commercialization should be tested for its effect on diabetic animals. There are several strategies to induce diabetes in mice, rats or other animal models. In this study, we demonstrated a safe method for producing diabetic condition in mice to be tested for anti-diabetic effect of any pharmaceutical drug. In general, diabetes in mice/rat modelsis induced by chemical drugs, such as alloxan and streptozotocin.

The majority of studies published in the field of ethnopharmacology between 1996 and 2006 employed these models. Streptozotocin (STZ, 69\%) and alloxan (31\%) are by far the most frequently used drugs, and this model has been useful for the study of multiple aspects of the disease. Both drugs exert their diabetogenic action when they are administered parenterally: intravenously, intraperitoneally or subcutaneously. Apart from this, while inducing diabetes in mice or rat models, sometimes rate of mortality is high, hence, the dose of these agents required for inducing diabetes depends on the animal species, route of administration and nutritional status. Therefore, here in the present study, we modified and observed the dose pattern of streptozotocin for safe diabetes induction in experimental mice before drug administration.

There are a large number of natural biomolecules that have been discussed in literature for their commercial and safer anti-diabetic effects. For example, plants have been used since ancient times to prevent conditions associated with diabetes (Soumyanath, 2003). The mechanism is most often not 
completely understood, however more and more studies are being conducted to elucidate the mechanisms of action of different plants and natural compounds.

Therefore, the object of this research is to show visual demonstration of diabetes induction in experimental mice model and to evaluate the pharmacological potency of any known and/or unknown drugs against diabetes prior to their clinical trial approval.

\section{MATERIALS AND EQUIPMENTS}

\section{Experimental animals}

Susceptible strain of 4 to 5 weeks old mice (male) of known weight

\section{Disposables}

Disposable syringe $(1 \mathrm{~mL})$

Cotton swab pads (70\% ethanol)

Surgical gloves

Surgical masks

Soft sterilized lancets

Sterilized distilled water

\section{Major equipments}

Cannula for oral drug administration

Glucometer and glucose test strips

Weighing machine

Paraformaldehyde (4\%) for tissue preservation

Freezer $\left(-70^{\circ} \mathrm{C}\right.$ for preservation of animal tissue slides until further analysis)

Streptozotocin (STZ) from Sigma

Pioglitazone (standard anti-diabetic drug)

Test drug sample

Ethanol

\section{PREPARATION OF REAGENTS}

\section{Citrate buffer solution 0.1M ( $p H$ 2.5)}

Citric acid (Sigma): $\quad 2.101 \mathrm{~g} / 100 \mathrm{~mL}$ distilled water

Sodium citrate (Sigma): $2.941 \mathrm{~g} / 100 \mathrm{~mL}$ distilled water

$46.5 \mathrm{~mL}$ of citric acid with $3.5 \mathrm{~mL}$ of sodium citrate solution

Make up to $100 \mathrm{~mL}$ in a volumetric flask.

This gives citrate buffer at $\mathrm{pH} 2.5$

\section{VIDEO CLIPS}

Handling and caring of mice

$2 \min 30 \mathrm{sec}$

Inducing diabetes in mice and observing blood glucose level

$1 \mathrm{~min} 47 \mathrm{sec}$

Drug administration and observation of blood glucose level

$2 \mathrm{~min} 11 \mathrm{sec}$ 


\section{METHOD}

\section{Experimental animal care}

1. Four to five weeks old ICR mice were purchased from Samtaco bio Korea (Osan, Korea).

2. Mice were divided in different cages $n=6$ in each group

3. Animal use protocol was approved by the Yeungnam University, South Korea and was in accordance with international standard on the care and use of experimental animals (CCAC, 1999).

4. Animals were housed under standard conditions of temperature $\left(25^{\circ} \mathrm{C}\right), 12$ hours $/ 12$ hours light/dark cycles under relative humidity $30-70 \%$ and fed with standard high fat pellet diet and tap water (for 2-3 weeks to get them adapted in new environment).

\section{Toxicity study}

1. To assess the toxicity, healthy mice used for study, starved overnight, kept in different groups $(n=6)$.

2. Orally fed with the known and/or unknown drug (For which we want to evaluate anti-diabetic potential).

3. The mice were observed continuously after 24 and 72 hours for any lethality (Turner, 1965).

[Pre-clinical development often includes pharmacology studies in which toxicology studies for antidiabetic therapies generally should be conducted in the standard non-diabetic animal models.]

\section{Induction of diabetes in mice}

1. Mice were kept on overnight fasting prior to induce diabetes.

2. Mice were treated twice with intraperitoneal injection $(200 \mu \mathrm{L})$ of streptozotocin $(100 \mathrm{mg} / \mathrm{kg}$ body weight) after overnight fasting.

3. Withdraw blood from the tail vein of mice and measure the blood glucose level in diabetes inducing mice of each group.

4. Again single dose of intraperitoneal injection $(200 \mu \mathrm{L})$ of streptozotocin $(100 \mathrm{mg} / \mathrm{kg}$ body weight $)$ after overnight fast.

5. Measure the diabetes level after 3 days of injection.

6. The mice with blood glucose level above $250 \mathrm{mg} / \mathrm{dL}$ were considered diabetic and were used for experimental study.

[Mice were routinely observed for loss of body weight]

\section{Observation of blood glucose levels in streptozotocin-induced diabetic mice}

1. All mice were grouped accordingly to the tested known and/or unknown drug, control (only water and feed) and standard antidiabetic drug.

2. Oral administration of test drug, and standard drug was performed until 6 weeks.

3. Withdraw blood from the tail vein of mice.

4. Measurement of blood glucose levelby using glucometer after each 4 th day.

[Mice were always observed for body weight]

[At the end of drug administration, all mice can be sacrificed and blood plasma and pancreatic tissues will go for further biochemical and histopathological analysis]

\section{DISCUSSION}

In the light of the results, our study indicates that the tested drug has anti-diabetic activity as confirmed by the visual demonstration and differences in the blood glucose levels of control and treatment mice. These results will allow for further human trial of drug before its commercialization. Diabetes mellitus of long duration is associated with several complications such as atherosclerosis, myocardial infarction, 
neuropathy and nephropathy. These complications have long been assumed to be related to chronically elevated glucose levels and subsequent oxidative stress. To provide information on underlying mechanisms and to evaluate potential therapies, experimental research on diabetic neuropathy is usually carried out using genetic or chemically induced diabetic animal models. An outline featuring the pharmacological effects of any proposed test drug for its commercialization serving as an anti-diabetic agent has been summarized in Figure 1.

A systemic administration of streptozotocin (STZ) has been reported to induce hyperalgesia to thermal, mechanical and chemical stimuli (Courteix et al., 1993). STZ-induced hyperalgesia is frequently associated with hyperglycemia because in some studies its development was prevented by insulin treatment (Aley and Levine, 2001). Although these studies suggest that STZ induces painful diabetic neuropathy, it is important to point out that the majority of studies evaluating STZ-induced hyperalgesia only include animals rendered hyperglycemic (Cunha et al., 2009). In the study carried out by Cunha et al. (2009), they reported that administration of high dose $(40 \mathrm{mg} / \mathrm{kg}$ body $\mathrm{wt})$ and low dose $(10 \mathrm{or} 20 \mathrm{mg} / \mathrm{kg}$ body wt) of streptozotocin produced mechanical hypernociception in all the STZ challenged rats whereas the low dose failed to produce hyperglycemia, suggesting that some other factor other than hyperglycemia could be involved in STZ-induced mechanical hypernociception. However, the mechanism of action of these antidiabetic drugs in reducing the diabetes is not known.

In fact, it has been found that natural phytomedicines used in human diabetes also have a significant antioxidant activity. Therefore, it may be possible that these phytomedicines may reduce the effect of inflammatory cytokines released during diabetes which may be one of the causative agents for the tissue distraction and insulin resistance (Shukla et al., 2011). Influence with the above findings, therefore, in the present study, we provided step-by-step protocol for inducing diabetes in animal mice model and to test our sample for proving its efficacy as anti-diabetic agent before its further pre-human trials and commercialization, which will certainly help in other vital aspects to control this autoimmune and widespread disease, providing sufficient personnel and resources for diabetes control in all healthcare facilities.

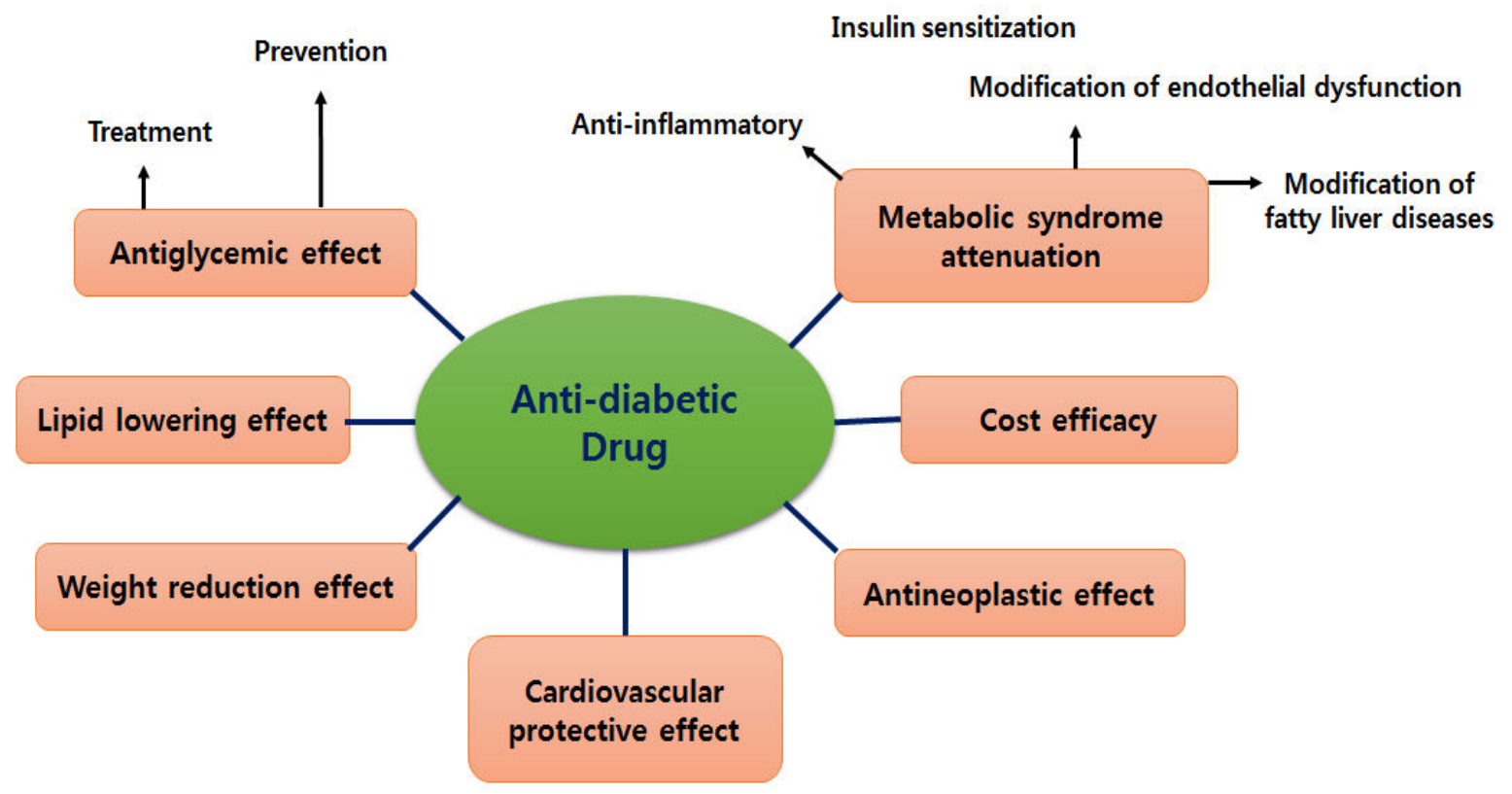

Figure 1: An outline featuring the pharmacological effects of any proposed test sample serving as an anti-diabetic drug

\section{REFERENCES}

Aley KO, Levine JD. Rapid onset pain induced by intravenous streptozotocin in the rat. J Pain. 2001; 2: 146-50.

CCAC. Guide to the care and used of experimental animals. Vol. 1. The Canadian Council on Animal Care, 1993. 
Courteix C, Eschalier A, Lavarenne J. Streptozotocin-induced diabetic rats: Behavioral evidence for a model of chronic pain. Pain 1993; 53: 81-88.

Cunha JM, Funez MI, Cunha FQ, Parada CA, Ferreira SH. Streptozotocin induced mechanical hypernociception is not dependent on hyperglycemia. Braz J Med Biol Res. 2009; 42: 197-206.

Frode TS, Medeiros YS. Animal models to test drugs with potential anti-diabetic activity. J Ethnopharmacol.2008; 115: 173-183.

Shukla S, Mehta P, Bajpai VK. Evaluation of comparative anti-diabetic effects of ethanolic extracts of Caesalpinia bouncucella and Stevia rebaudiana in normal and alloxan-induced experimental rats. Rom Biotechnol Lett. 2011; 16: 6187-99.

Soumyanath A. Traditional medicines for modern times: Anti-diabetic plants. London, CRC Press, 2006.

Turner MA. Screening methods in pharmacology. New York, Academic Press, 1965, p 26.

\section{PRECAUTION}

While inducing mice for diabetes, we should consider reduced mortality rate due to high dose of streptozotocin.

Animals should be handled according to handling ethical rules.

Animals should not be stressed during drug administration and observation as that can make effect on metabolic conditions of animals.

Avoid doing following things such as injection, drug administration and measurement of blood glucose on same day in order to avoid stress situation.

Use of laboratory hand gloves and 70\% ethanol should be maintained to avoid any cross contamination and injury. 


\section{Your feedback about this paper}

1. Number of times you have read this paper 0

2. Number of times you have seen the video clip 0

3. Which video you may need to see again, if any

4. Quality of paper
O Excellent
O Good
O Moderate
Not good

5. Your comments

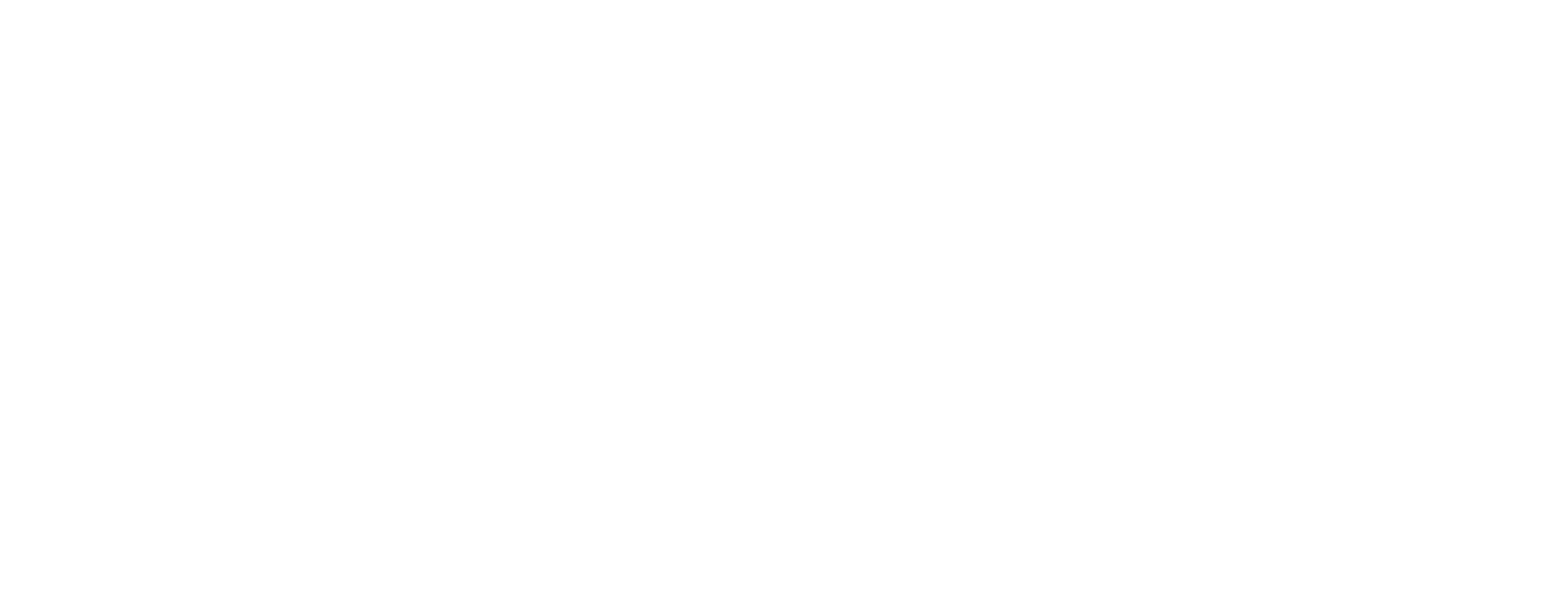

